\title{
Applying Propensity Score Methods in Clinical Research in Neurology
}

Peter C. Austin, PhD, Amy Ying Xin Yu, MD, MSc, Manav V. Vyas, MD, PhD, and Moira K. Kapral, MD, MSc Neurology ${ }^{\circledR}$ 2021;97:856-863. doi:10.1212/WNL.0000000000012777
Correspondence

Dr. Austin

peter.austin@ices.on.ca

\begin{abstract}
Propensity score-based analysis is increasingly being used in observational studies to estimate the effects of treatments, interventions, and exposures. We introduce the concept of the propensity score and how it can be used in observational research. We describe 4 different ways of using the propensity score: matching on the propensity score, inverse probability of treatment weighting using the propensity score, stratification on the propensity score, and covariate adjustment on the propensity score (with a focus on the first 2). We provide recommendations for the use and reporting of propensity score methods for the conduct of observational studies in neurologic research.
\end{abstract}

\section{Introduction}

Observational studies are increasingly being used to estimate the effects of treatments and exposures. Such studies can often be conducted rapidly and at less expense than randomized controlled trials (RCTs) and can estimate the effects of treatments as they are used in real-world clinical practice. Furthermore, they permit the study of exposures for which it would be unethical to randomize patients (e.g., tobacco smoking), or, in some cases, not possible to randomize (e.g., sex). However, in observational studies, unlike in RCTs, treated participants (used hereafter for exposed participants) frequently differ at baseline from those receiving the control intervention. Studies are said to be subject to confounding when the distribution of variables that influence the outcome differs between treated and control participants. In the presence of confounding, differences in outcomes between treatment groups may be due, at least in part, to these systematic differences between treated and control participants.

In observational studies, statistical methods are required to reduce the effects of confounding when estimating the effects of treatments. Methods based on the propensity score are increasingly being used for this purpose. The objective of this article is to introduce the propensity score and its application to neurologic research. We define the propensity score, describe how it can be estimated in an observational cohort study, and review different methods for using the propensity score.

\section{Two Types of Questions}

There are 2 common questions that one can ask when estimating the effect of a treatment. First, what is the effect of treatment in the entire population or sample of eligible patients? This is equivalent to asking how outcomes would differ if everyone received the treatment of interest compared to if everyone received the control treatment. Second, what is the effect of treatment in those patients who ultimately received the treatment of interest? This is equivalent to asking how outcomes would differ in the treated participants if, contrary to what occurred, they had not been treated. The former question is about the average treatment effect (ATE), while the latter is about 


\section{Glossary}

ATE $=$ average treatment effect; ATT $=$ average treatment effect in the treated; IPTW $=$ inverse probability of treatment weighting; NNM = nearest neighbor matching; NNT = number needed to treat; RCT $=$ randomized controlled trial; $\mathbf{t P A}=$ tissue-type plasminogen activator.

the average treatment effect in the treated (ATT). Understanding the type of question that one is asking will help determine the most appropriate propensity score method to use.

In many pharmacoepidemiologic studies, the ATE may be of greater interest, as one can think of everyone in the eligible population as being treated with either agent. However, the ATT may be of greater interest in settings in which, despite all participants being eligible for either treatment, there are barriers or burdens imposed by one of the treatments. For example, if the cost of a drug is not routinely covered by insurance, it may make more sense to estimate the effect of this drug in those participants who ultimately decide to pay for it out of pocket.

\section{Defining and Estimating the Propensity Score}

We assume an observational cohort study design with a binary treatment (treated vs control) that is assessed at baseline (i.e., time of cohort entry). Treatment can either involve 2 active treatments (e.g., natalizumab vs another diseasemodifying therapy for patients with multiple sclerosis) or an active treatment compared with a null control treatment (e.g., edavarone vs standard care in patients with amyotrophic lateral sclerosis). Baseline characteristics are measured on each participant. The outcome is measured after cohort entry (and implicitly after the treatment has been applied). Outcomes can be of types that are commonly seen in clinical research: continuous (e.g., blood pressure), binary (e.g., disabled on discharge vs not disabled on discharge), time-to-event (e.g., time to death), or a count (e.g., number of health care encounters). Any outcome that one can use in an RCT can be used in a study that uses propensity score methods.

The propensity score is defined as the probability of receiving the treatment of interest (vs the control treatment) conditional on measured participant covariates. ${ }^{1,2}$ The primary property of the propensity score is that it is a balancing score. ${ }^{1}$ This means that in a subgroup of participants, all of whom have the same value of the propensity score, the distribution of measured baseline covariates will be the same in treated and control participants in that subgroup. Thus, we can remove the effects of confounding by comparing outcomes between treated and control participants who share a similar value of the propensity score. This balancing is analogous to that induced by randomization in RCTs, with the key difference being that conditioning on the propensity score balances measured covariates, whereas randomization ideally balances both measured and unmeasured covariates. Note that balance is a large sample property, and that a sufficiently large sample is necessary in order to expect to observe balance in a given sample.

The validity of conclusions drawn from an analysis that uses propensity score methods rests on 2 assumptions: (1) the assumption that the investigators have measured all confounding variables and that there are no unmeasured confounders and (2) the positivity assumption that each participant has a nonzero probability of receiving each treatment. ${ }^{1}$ This implies that no participant has an absolute contraindication to either the active or control treatment. Participants with an absolute (or relative) contraindication to either treatment should be excluded at the design stage, as is done in the design of RCTs. We refer the reader elsewhere for a discussion of positivity and methods to identify its presence. ${ }^{3}$ As an example, in a study comparing outcomes between immigrants to Ontario and long-term residents, participants residing in rural areas were excluded because, in Ontario, immigrants reside almost exclusively in urban areas. ${ }^{4}$ These assumptions are no different from those required by other methods for estimating causal effects in observational studies.

The propensity score is frequently estimated using a logistic regression model in which treatment status (treatment vs control) is regressed on the measured baseline covariates. While logistic regression is the most frequently used approach, researchers can also use algorithmic approaches from the machine learning literature, such as random forests or generalized boosting models. Strategies for variable selection for the propensity score model have been described elsewhere; see the Table for a summary of 2 strategies that perform well. ${ }^{5}$

Once the propensity score has been estimated, there are 4 main ways of using it: matching on the propensity score, inverse probability of treatment weighting (IPTW) using the propensity score, stratification on the propensity score, and covariate adjustment using the propensity score.

\section{Matching on the Propensity Score}

Matching on the propensity score entails forming matched sets of treated and control participants who share a similar value of the propensity score. The most common implementation is pair-matching, in which pairs of treated and control participants are formed. There are 2 common implementations of pairmatching. The first is greedy nearest neighbor matching 
(NNM), in which a treated participant is selected at random and then matched to the control participant whose propensity score is closest to that of the treated participant. The process is described as greedy because at each stage the control is selected who is closest to the currently considered treated participant, even if that untreated participant would serve better as a control for a subsequent treated participant. This process is then repeated until a matched control participant has been selected for each treated participant. This process generally uses matching without replacement, so that once a control participant is matched to a treated participant, that control participant is no longer available for matching to a subsequent treated participant. A refinement to NNM is NNM with a caliper restriction. Using this approach, a control participant is an acceptable match for a treated participant only if the difference in their propensity scores is less than a maximum amount (the caliper width or distance). For technical reasons, one typically matches on the logit of the propensity score and uses a caliper width that is defined as a proportion of the SD of the logit of the propensity score. ${ }^{6}$ Prior research has shown that a caliper of width equal to 0.2 of the SD of the logit of the propensity score works well in a variety of settings. ${ }^{7}$ Readers are referred elsewhere for a discussion and examination of different matching algorithms. ${ }^{8,9}$ An alternative to greedy matching is optimal matching, which forms matched pairs to minimize the total within-pair difference in the propensity score. Alternative matching algorithms to pair-matching have been described in the literature. These include fixed ratio M:1 matching, variable ratio matching, and full matching. ${ }^{10-15}$ While NNM with a caliper restriction is often a preferred approach, an attraction of these alternative matching algorithms is the ability to include a larger number of control participants in the analytic sample.

Matching on the propensity score can be combined with exact matching on a small number of baseline covariates. This will ensure perfect balance on these variables between treated and control participants in the matched sample. This approach is important if one wants to conduct subsequent subgroup analyses, so that matched pairs are included in the same subgroup. If one wanted to conduct subsequent sex-specific analyses, one could match on the propensity score and on sex, so that matched participants were of the same sex. When matching on a variable such as sex, it is not necessary that that variable be excluded from the propensity score model. Matching on the propensity score does not guarantee that matched participants will be identical on all measured variables. Rather, the distribution of the covariates will be similar between treated and control participants in the matched sample.

A crucial step in any study that uses propensity score matching is to assess the degree to which matching on the propensity score resulted in the formation of a matched sample in which the distribution of baseline characteristics is similar between treated and control participants. This assessment is critical as it allows both the researcher and readers to assess whether matching on the estimated propensity score has removed systematic baseline differences between treatment groups. This assessment should always be conducted while blinded to the outcomes (i.e., before any comparison of outcomes between treated and control participants). The use of statistical significance testing for assessing differences in baseline characteristics has been criticized by different sets of authors. ${ }^{16,17}$ Instead, we recommend the use of statistics that are properties of samples and that, unlike statistical hypothesis testing, do not refer to hypothetical superpopulations. The use of the standardized difference, which is the difference in means in units of SD, is often used for assessing the similarity of matched treated and control participants. Some authors have suggested that a threshold of 0.10 (or 10\%) be used to denote acceptable balance after matching. ${ }^{18}$ While most researchers limit balance assessment to using standardized differences to compare the means and prevalences of covariates between treatment groups, a comprehensive suite of balance diagnostics is described elsewhere for those wanting to conduct a more extensive assessment. ${ }^{19}$ If inadequate balance is observed, the researchers are encouraged to modify the specification of the propensity score model. Possible modifications include allowing for nonlinear relationships between continuous covariates and the log-odds of treatment (e.g., using restricted cubic splines) or the inclusion of interaction terms. ${ }^{20}$ For example, Rosenbaum and Rubin, ${ }^{20}$ in the first application of propensity score methods, described an iterative process that required 4 iterations to achieve acceptable balance.

Once acceptable balance has been achieved, analysts can unblind themselves to the outcome and compare outcomes between treated and control participants in the matched sample. The analyses conducted in the propensity scorematched sample can be similar to those that would be done in an RCT with a similar outcome. Thus, if the outcome is continuous (e.g., the Unified Parkinson's Disease Rating Scale), one can compute the mean blood pressure in each treatment group and then compute the difference in mean blood pressure. Similarly, if the outcome is binary (e.g., resolution of symptoms), one can compute the proportion of successes in each treatment group. One can then compute the absolute risk reduction (difference in the estimated

Table Strategies for Selecting Variables to Include in a Propensity Score Model

\begin{tabular}{lll}
\hline & $\begin{array}{l}\text { (A) Include all variables } \\
\text { prognostic for the outcome }\end{array}$ & $\begin{array}{l}\text { (B) Include only } \\
\text { confounding variables }\end{array}$ \\
\hline Definition & $\begin{array}{l}\text { All prognostically important } \\
\text { variables, regardless of } \\
\text { whether they are also } \\
\text { associated with treatment } \\
\text { assignment }\end{array}$ & $\begin{array}{l}\text { Variables that are associated } \\
\text { with both the treatment } \\
\text { assignment and the outcome }\end{array}$ \\
\hline Comments & $\begin{array}{l}\text { Easier to implement; } \\
\text { prognostically important } \\
\text { variables likely to be constant } \\
\text { across jurisdictions and can } \\
\text { be identified from the } \\
\text { literature }\end{array}$ & $\begin{array}{l}\text { Represents a subset of all } \\
\text { prognostically important } \\
\text { be influenced by regulations, } \\
\text { insurance, local practice, etc, } \\
\text { and thus may vary across } \\
\text { jurisdictions }\end{array}$ \\
\hline
\end{tabular}


proportions in each treatment group) and the relative risk (ratio of the estimated proportions in each treatment group). From the former, one can estimate the number needed to treat (NNT), which some have described as a key statistic for medical decision-making. ${ }^{21,22}$ Note that there is no need to report an odds ratio. If the outcome is time-to-event in nature, one can estimate Kaplan-Meier survival curves (or cumulative incidence functions if there are competing risks) in treated and control participants. ${ }^{23,24}$ These survival curves can be complemented by estimating a univariate Cox model (or causespecific hazard model if there are competing risks) in which the hazard of the outcome is regressed on treatment status.

While estimation of the effect of treatment (e.g., estimating the hazard ratio or risk difference) can be done using analyses that reflect those that would be conducted in a similar RCT, the standard error of the estimated effect (used for constructing confidence intervals) should account for the matched nature of the sample. ${ }^{25-28}$ Thus, for instance, with a Cox model, a robust variance estimator can be used, ${ }^{26}$ while one can use the McNemar test for comparing proportions between groups. ${ }^{27}$

When using matching on the propensity score, one is estimating the ATT. One cannot make inferences about the effect of treatment in the entire population of treated and control participants.

\section{Propensity Score Matching in the Neurology Literature}

$\mathrm{Yu}$ and colleagues ${ }^{29}$ used matching on the propensity score to estimate the efficacy of IV thrombolysis with recombinant tissue-type plasminogen activator (tPA) for acute ischemic stroke in routine clinical practice. In order to account for baseline differences between groups, they used propensity score matching using NNM caliper matching with a caliper of 0.2 times the SD of the logit of the propensity score. The authors used standardized differences to confirm that there were no meaningful differences in measured baseline covariates between treated and control participants in the matched sample. Having satisfied themselves that matching had substantially decreased differences between the 2 groups, the authors compared mortality in those who did and did not receive tPA using Kaplan-Meier survival curves (compared using a stratified log-rank test to account for the matched nature of the sample) and a Cox proportional hazards model to compare the hazard of death between treated and control participants.

An advantage to using propensity score matching in this context is that it discards those untreated participants who are dissimilar to any treated participant and retains only controls who are similar to the treated participants. Yu and colleagues ${ }^{29}$ estimated the effect of tPA in those who received it. Some applied researchers are wary of using propensity score matching for fear of excluding participants from the matched sample, but this concern is generally unwarranted. It is important to identify a matched control for each treated participant, as failure to do so can result in bias due to incomplete matching (because one is trying to estimate the effect of treatment in only a subset of treated participants who may differ systematically from the overall population of treated participants). However, once a control participant has been identified for each treated participant, the remaining unused control participants are superfluous and their exclusion from the analysis is unimportant. For this reason, matching works best in those settings in which there are substantially more controls than treated participants, as this increases the likelihood of identifying a suitable control for each treated participant.

\section{IPTW Using the Propensity Score}

IPTW using the propensity score creates weights based on the propensity score. ${ }^{30}$ In the weighted sample, the distribution of measured baseline covariates will be the same in treated participants as in control participants. Thus, the presence of confounding is removed by weighting and outcomes can be compared directly between treated and control participants in the weighted sample. IPTW can be understood through the lens of complex surveys that incorporate sampling weights. Just as survey weights allow one to standardize the survey sample so that it is reflective of a given population, the use of IPTW standardizes each of the treated and control samples to a common reference sample.

Let $\mathrm{Z}$ be a binary variable denoting treatment status $(\mathrm{Z}=1$ for treated vs $\mathrm{Z}=0$ for control) and $e$ denote the propensity score. Conventional inverse probability of treatment weights are defined as $w=\frac{Z}{e}+\frac{1-Z}{1-e}$. Thus, all participants are weighted by the reciprocal of the probability of receiving the treatment that they actually received. This set of weights uses the combined sample of treated and control participants as the target population to which each is standardized. A modification of these weights is stabilized weights: $w_{\text {stab }}=\operatorname{Pr}(Z=1) \frac{Z}{e}+\operatorname{Pr}(Z=0) \frac{1-Z}{1-e^{.}} \cdot{ }^{31}$ The use of stabilized weights may reduce the effect of a small number of participants with very high weights, which can result in improved variance estimation. The use of either conventional inverse probability of treatment weights or stabilized weights allows the investigator to estimate the ATE. An alternative set of weights allows one to estimate the ATT: $w_{\text {att }}=Z+\frac{e(1-Z)}{1-e} \cdot 32,33$ Thus, treated participants have a weight of 1 , while control participants have a weight of $\mathrm{e} /(1-\mathrm{e})$. This implies that the treated participants are the reference population to which each of the treated and control samples are standardized. The investigator must choose the set of weights (ATE vs ATT) that is appropriate to address the specific study question. While ATE and ATT weights are the most commonly used propensity score-based weights, matching weights, overlap weights, and entropy weights are alternatives. ${ }^{34}$ 
Once the weights have been estimated, it is important to examine the distribution of the weights, as very large weights can affect variance estimation of the treatment effect and participants with very large weights can exert undue influence on the analyses and result in unstable estimates. There is no definition as to what constitutes a large weight. Strategies to address the presence of large weights include trimming large weights, so that weights that exceed a given threshold are truncated to equal this threshold (e.g., weights that exceed the 99th percentile of weights are set equal to the 99th percentile) or excluding participants whose propensity score is less than 0.1 or greater than $0.9 .^{35}$ Additional weight-based diagnostics are described elsewhere. ${ }^{36}$

Once the propensity score has been estimated and the appropriate set of weights has been constructed, an assessment of the degree to which using the weights has allowed one to balance measured baseline covariates between treatment groups must be conducted prior to examining the effect of treatment on outcomes. An extensive set of balance diagnostics for use with propensity score weighting has been described elsewhere. ${ }^{36}$ As with matching, the use of standardized differences in the weighted sample is recommended and the process of specifying the propensity score can proceed iteratively until acceptable balance is achieved.

Because weighting has removed the effects of confounding, one can estimate the effect of treatment using methods similar to those used in a comparable RCT. For continuous outcomes, one can estimate the weighted mean outcome in treated and control participants separately and then compute the difference in means. For binary outcomes, one can estimate the weighted proportion of successes in treated and control participants and then compute an absolute risk reduction, a relative risk, and an NNT. As with matching, there is no need to estimate an odds ratio. With time-to-event outcomes, weighted Kaplan-Meier survival curves (or cumulative incidence functions in the presence of competing risks) can be estimated, along with a weighted Cox proportional hazards model. Lunceford and Davidian ${ }^{37}$ describe methods to estimate the variance of differences in means (which can also be used with risk differences). Xie and $\mathrm{Liu}^{38}$ describe a test for equality of weighted survival curves. When using a regression model (e.g., a Cox model) in the weighted sample, variance estimation must account for the within-participant homogeneity induced by the weights. ${ }^{39,40}$ One option is to use a robust variance estimator. ${ }^{26}$ For all estimated measures of effect (e.g., a relative risk or a hazard ratio), one can use bootstrapping to construct confidence intervals. When using the bootstrap, it would be important to estimate the propensity score within each bootstrap sample in order to reflect the variability in the estimated statistic.

\section{IPTW in the Neurology Literature}

Vyas and colleagues ${ }^{4}$ used IPTW to compare mortality after ischemic stroke between immigrants and long-term residents.
They used standardized differences to compare baseline covariates between immigrants and long-term residents in the weighted sample and confirmed that these were $<0.10$. Both Kaplan-Meier survival curves and a Cox regression model were used to compare mortality between immigrants and long-term residents in the weighted sample.

Hersh and colleagues ${ }^{41}$ used IPTW to compare relapse between switching from natalizumab to a moderate-efficacy disease-modifying therapy vs high-efficacy therapy in patients with multiple sclerosis. The authors used ATT weights with those switching to a moderate-efficacy disease-modifying therapy as the reference population. The authors used standardized differences to assess the balance in baseline covariates induced by weighting; after weighting, all but 5 of the standardized differences were less than $10 \%$. The authors then used a Poisson regression model to compare the relapse rate between treatment groups.

\section{Stratification and Covariate Adjustment Using the Propensity Score}

Stratification on the propensity score involves ranking participants on the estimated propensity score and then dividing the sample into approximately equal size strata based on specified percentiles of the propensity score. Using 5 strata based on quintiles of the propensity score is the most common approach in practice. One then compares treated and control participants in each stratum and computes stratumspecific estimates of treatment effect, which are then pooled to generate an overall estimate of treatment effect.

Covariate adjustment using the propensity score involves regression of the outcome on an indicator variable denoting treatment status and the propensity score. This method was initially suggested for use with continuous outcomes and when estimating a linear treatment effect (e.g., a difference in means). However, it was subsequently frequently applied in settings with binary or time-to-event outcomes.

Lunceford and Davidian ${ }^{37}$ suggest that stratification is affected by "biased inference due to residual confounding, and the effect of this bias becomes more serious with increasing sample size". Other research has shown that both stratification on the propensity score and covariate adjustment using the propensity score can lead to biased estimation of odds ratios and hazard ratios, ${ }^{26,42,43}$ and that covariate adjustment using the propensity score resulted in a greater magnitude of residual confounding than did matching on the propensity score or IPTW. ${ }^{44}$ A further limitation of covariate adjustment using the propensity score is that it relies on a regression model to relate the outcome to treatment status and other variables (the propensity score). This approach requires the assumption that the outcomes regression model has been correctly specified. 


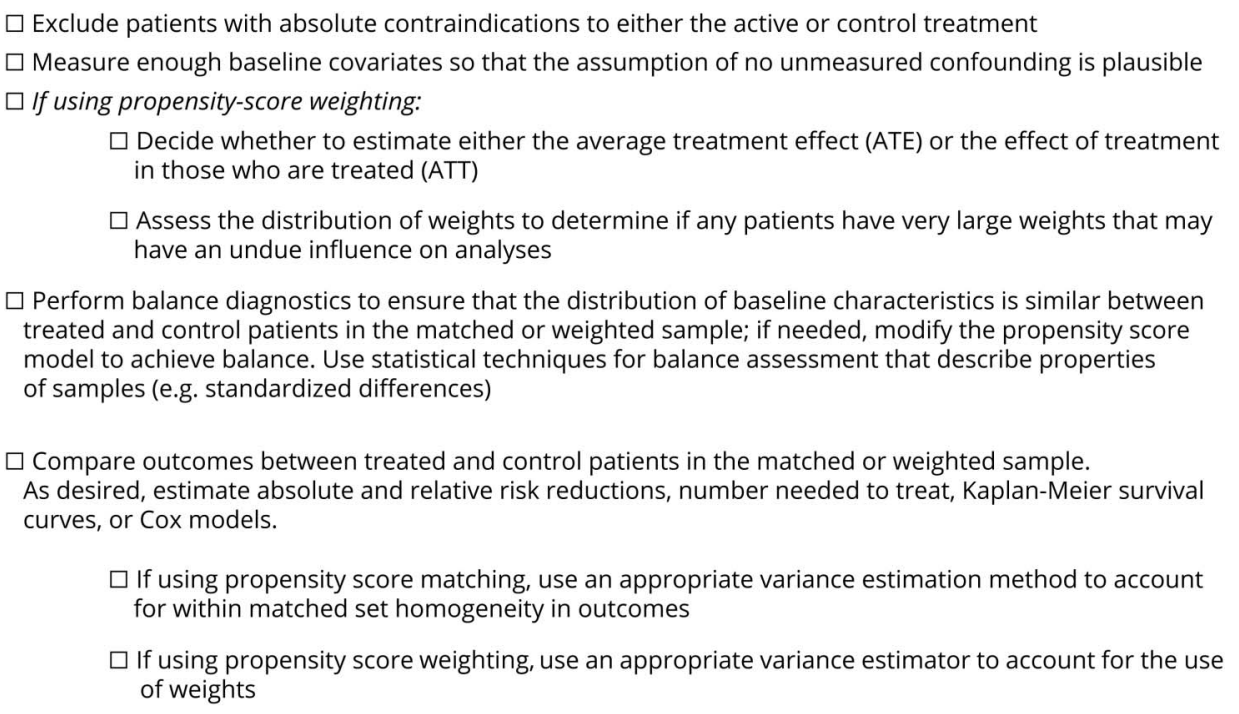

For these reasons, while these 2 propensity score methods may be preferable in specific settings, we generally encourage researchers to consider either matching or weighting as the first propensity score approach to be considered.

\section{Using the Propensity Score to Estimate Effects of Nonbinary Treatments}

In the preceding sections we have focused on methods to use the propensity score to estimate the effects of binary treatment (treated vs control), as this is the most common application of propensity score methods. The generalized propensity score is an extension of propensity score methods to continuous or quantitative treatments (e.g., number of antiepileptic medications). ${ }^{45-48}$ When considering a nonbinary categorical treatment (e.g., glatiramer acetate vs interferon- $\beta 1 \mathrm{~b}$ vs interferon- $\beta$ 1a for treatment of multiple sclerosis), one possible approach is to conduct a sequence of analyses, each consisting of a comparison of 2 of the different levels of the treatment. Alternatively, methods using a multinomial logistic regression model to estimate the propensity score have been described. ${ }^{49}$ Further research is necessary to determine the relative performance of these approaches.

\section{Propensity Score vs Regression- Based Methods}

Many researchers are familiar with regression-based approaches to account for confounding and may wonder whether it is worthwhile to learn a new technique. There are several advantages to the use of propensity score matching

Figure 2 Author Checklist for the Reporting of Studies Using Propensity Score Methods

$\square$ List the variables included in the propensity-score model and the rationale for their inclusion

$\square$ If propensity-score matching was used:

$\square$ Explicitly describe the matching method (e.g. greedy nearest neighbor matching, caliper restriction, optimal matching, etc.) to allow others to replicate study findings

$\square$ List any covariates on which an exact match was forced

$\square$ Report the percentage of treated patients for whom a control patient was identified

$\square$ Present the results of balance diagnostics. Include a table or figure comparing measured baseline covariates in treated and control patients in the matched sample

$\square$ If propensity-score weighting was used:

$\square$ State the type of weight used (e.g. stabilized IPTW weights) to allow others to replicate the study findings

$\square$ Present results of balance diagnostics. Include a table or figure comparing measured baseline covariates in treated and control patients in the weighted sample 
and weighting over conventional regression adjustment. First, when outcomes are binary, one can report absolute risk differences, relative risks, and NNTs. Similarly, with time-toevent outcomes, one can report absolute differences in survival at specific time points (obtained from the estimated survival curves) and hazard ratios. In contrast, with conventional regression adjustment, one is generally limited to reporting odds ratios and hazard ratios. Although the other measures can be estimated with additional work, this is rarely done in practice. Second, through describing the distribution of baseline covariates in treated and control participants in the matched or weighted sample, one can illustrate the degree to which matching or weighting has removed systematic baseline differences between treatment groups. It is much more difficult to determine the degree to which regression adjustment has minimized differences between groups. Finally, in settings in which outcomes are rare and the sample size is low to moderate, regression-based approaches would be limited in terms of the number of covariates that could be included in the regression model. This limitation does not apply to propensity score methods.

\section{Limitations of Propensity Score Methods}

Propensity score methods allow one to remove the effects of confounding due to measured baseline covariates. They make no claim to remove the effects of confounding due to unmeasured covariates, and findings from observational studies must be interpreted with care given this potential for residual confounding (a limitation shared by regression adjustment). Rosenbaum ${ }^{50}$ described sensitivity analyses that can be used with propensity score matching to assess the robustness of study conclusions to unmeasured confounding.

\section{Summary}

Propensity score methods reduce the effects of confounding due to measured baseline covariates by creating a matched or weighted sample in which the distribution of measured baseline covariates is similar in treated and control participants. This allows for a direct comparison of outcomes between treatment groups and the use of metrics of treatment effect similar to what would be done in a comparable RCT. We have provided a checklist of study design and analysis considerations in Figure 1 and a reporting checklist for manuscripts that use propensity score matching or weighting in Figure 2.

We hope this brief introduction will be of use to neurologic researchers and that the checklists and recommendations provided will strengthen the quality of observational studies that use propensity score methods.

\section{Acknowledgment}

This study was supported by ICES, which is an independent, nonprofit research institute funded by an annual grant from the Ontario Ministry of Health (MOH) and the Ministry of LongTerm Care (MLTC). The opinions, results, and conclusions reported in this article are those of the authors and are independent from the funding sources. No endorsement by ICES or the Ontario MOH or MLTC is intended or should be inferred.

\section{Study Funding}

This research was supported by an operating grant from the Canadian Institutes of Health Research (CIHR) (PJT 166161). Drs. Austin and Kapral are supported by MidCareer Investigator awards from the Heart and Stroke Foundation of Ontario. Dr. Kapral holds the Lillian Love Chair in Women's Health at the University Health Network/ University of Toronto. Dr. Yu is supported by a national new investigator award from the Heart and Stroke Foundation of Canada.

\section{Disclosure}

The authors report no disclosures relevant to the manuscript. Go to Neurology.org/N for full disclosures.

\section{Publication History}

Received by Neurology May 4, 2021. Accepted in final form August 9, 2021.

\begin{tabular}{|c|c|c|}
\hline Name & Location & Contributions \\
\hline $\begin{array}{l}\text { Peter C. } \\
\text { Austin, } \\
\text { PhD }\end{array}$ & $\begin{array}{l}\text { ICES, Toronto; Institute of } \\
\text { Health Management, Policy } \\
\text { and Evaluation, Department } \\
\text { of Medicine, University of } \\
\text { Toronto; Sunnybrook } \\
\text { Research Institute, Toronto, } \\
\text { Canada }\end{array}$ & $\begin{array}{l}\text { Drafting/revision of the } \\
\text { manuscript for content, } \\
\text { including medical writing for } \\
\text { content; study concept or } \\
\text { design }\end{array}$ \\
\hline $\begin{array}{l}\text { Amy Ying } \\
\text { Xin Yu, } \\
\text { MD, MSc }\end{array}$ & $\begin{array}{l}\text { ICES, Toronto; Divisions of } \\
\text { Neurology, Department of } \\
\text { Medicine, University of } \\
\text { Toronto }\end{array}$ & $\begin{array}{l}\text { Drafting/revision of the } \\
\text { manuscript for content, } \\
\text { including medical writing for } \\
\text { content }\end{array}$ \\
\hline $\begin{array}{l}\text { Manav V. } \\
\text { Vyas, MD, } \\
\text { PhD }\end{array}$ & $\begin{array}{l}\text { ICES, Toronto; Divisions of } \\
\text { Neurology, Department of } \\
\text { Medicine, University of } \\
\text { Toronto }\end{array}$ & $\begin{array}{l}\text { Drafting/revision of the } \\
\text { manuscript for content, } \\
\text { including medical writing for } \\
\text { content }\end{array}$ \\
\hline $\begin{array}{l}\text { Moira K. } \\
\text { Kapral, } \\
\text { MD, MSc }\end{array}$ & $\begin{array}{l}\text { ICES, Toronto; Institute of } \\
\text { Health Management, Policy } \\
\text { and Evaluation, Department } \\
\text { of Medicine, University of } \\
\text { Toronto; General Internal } \\
\text { Medicine }\end{array}$ & $\begin{array}{l}\text { Drafting/revision of the } \\
\text { manuscript for content, } \\
\text { including medical writing for } \\
\text { content; study concept or } \\
\text { design }\end{array}$ \\
\hline
\end{tabular}

\section{References}

1. Rosenbaum PR, Rubin DB. The central role of the propensity score in observational studies for causal effects. Biometrika. 1983;70:41-55.

2. Austin PC. An introduction to propensity-score methods for reducing the effects of confounding in observational studies. Multivariate Behav Res. 2011;46(3):399-424.

3. Westreich D, Cole SR. Invited commentary: positivity in practice. Am J Epidemiol. 2010;171(6):674-677; discussion 678-681. 
4. Vyas MV, Austin PC, Fang J, Laupacis A, Silver FL, Kapral MK. Immigration status, ethnicity, and long-term outcomes following ischemic stroke. Neurology. 2021;96(8) e1145-e1155.

5. Austin PC, Grootendorst P, Anderson GM. A comparison of the ability of different propensity score models to balance measured variables between treated and untreated participants: a Monte Carlo study. Stat Med. 2007;26(4):734-753.

6. Rosenbaum PR, Rubin DB. Constructing a control group using multivariate matched sampling methods that incorporate the propensity score. Am Statistician. 1985;39:33-38.

7. Austin PC. Optimal caliper widths for propensity-score matching when estimating differences in means and differences in proportions in observational studies. Pharm Stat. 2011;10:150-161.

8. Austin PC. Some methods of propensity-score matching had superior performance to others: results of an empirical investigation and Monte Carlo simulations. Biometrical J. 2009;51(1):171-184.

9. Austin PC. A comparison of 12 algorithms for matching on the propensity score. Stat Med. 2014;33(6):1057-1069.

10. Austin PC. Statistical criteria for selecting the optimal number of untreated subjects matched to each treated subject when using many-to-one matching on the propensity score. Am J Epidemiol. 2010;172(9):1092-1097.

11. Gu XS, Rosenbaum PR. Comparison of multivariate matching methods: structures, distances, and algorithms. J Comput Graphical Stat. 1993;2:405-420.

12. Ming K, Rosenbaum PR. Substantial gains in bias reduction from matching with a variable number of controls. Biometrics. 2000;56(1):118-124.

13. Rosenbaum PR. A characterization of optimal designs for observational studies. $J R$ Stat Soc Ser B. 1991;53:597-610.

14. Austin PC, Stuart EA. Optimal full matching for survival outcomes: a method that merits more widespread use. Stat Med. 2015;34(30):3949-3967.

15. Austin PC, Stuart EA. Estimating the effect of treatment on binary outcomes using full matching on the propensity score. Stat Methods Med Res. 2017;26(6):2505-2525.

16. Imai K, King G, Stuart EA. Misunderstandings between experimentalists and observationalists about causal inference. J R Stat Soc Ser A. 2008;171:481-502.

17. Austin PC. A critical appraisal of propensity-score matching in the medical literature between 1996 and 2003. Stat Med. 2008;27(12):2037-2049.

18. Mamdani M, Sykora K, Li P, et al. Reader's guide to critical appraisal of cohort studies: 2: assessing potential for confounding. Br Med J. 2005;330(7497):960-962.

19. Austin PC. Balance diagnostics for comparing the distribution of baseline covariates between treatment groups in propensity-score matched samples. Stat Med. 2009; 28(25):3083-3107.

20. Rosenbaum PR, Rubin DB. Reducing bias in observational studies using subclassification on the propensity score. J Am Stat Assoc. 1984;79:516-524.

21. Laupacis A, Sackett DL, Roberts RS. An assessment of clinically useful measures of the consequences of treatment. New Engl J Med. 1988;318:1728-1733.

22. Cook RJ, Sackett DL. The number needed to treat: a clinically useful measure of treatment effect. Br Med J. 1995;310(6977):452-454.

23. Austin PC, Schuster T. The performance of different propensity score methods for estimating absolute effects of treatments on survival outcomes: a simulation study. Stat Methods Med Res. 2016;25(5):2214-2237.

24. Austin PC, Fine JP. Propensity-score matching with competing risks in survival analysis. Stat Med. 2019;38(5):751-777.

25. Austin PC. Type I error rates, coverage of confidence intervals, and variance estimation in propensity-score matched analyses. Int J Biostatistics. 2009;5(1).

26. Austin PC. The performance of different propensity score methods for estimating marginal hazard ratios. Stat Med. 2013;32(16):2837-2849.

27. Austin PC. Comparing paired vs non-paired statistical methods of analyses when making inferences about absolute risk reductions in propensity-score matched samples. Stat Med. 2011;30(11):1292-1301.
28. Gayat E, Resche-Rigon M, Mary JY, Porcher R. Propensity score applied to survival data analysis through proportional hazards models: a Monte Carlo study. Pharm Stat. $2012 ; 11(3): 222-229$.

29. Yu AYX, Fang J, Kapral MK. One-year home-time and mortality after thrombolysis compared with nontreated patients in a propensity-matched analysis. Stroke. 2019, 50(12):3488-3493.

30. Rosenbaum PR. Model-based direct adjustment. J Am Stat Assoc. 1987;82:387-394.

31. Robins JM, Hernan MA, Brumback B. Marginal structural models and causal in ference in epidemiology. Epidemiology. 2000;11(5):550-560.

32. Morgan SL, Winship C. Counterfactuals and Causal Inference: Methods and Principles for Social Research. Cambridge University Press; 2007.

33. Sato T, Matsuyama Y. Marginal structural models as a tool for standardization. Epidemiology. 2003;14(6):680-686.

34. Zhou Y, Matsouaka RA, Thomas L. Propensity score weighting under limited overlap and model misspecification. Stat Methods Med Res. 2020;29(12):3721-3756.

35. Crump RK, Hotz VJ, Imbens GW, Mitnik OA. Dealing with limited overlap in estimation of average treatment effects. Biometrika. 2009;96(1):187-199.

36. Austin PC, Stuart EA. Moving towards best practice when using inverse probability of treatment weighting (IPTW) using the propensity score to estimate causal treatment effects in observational studies. Stat Med. 2015;34:3661-3679.

37. Lunceford JK, Davidian M. Stratification and weighting via the propensity score in estimation of causal treatment effects: a comparative study. Stat Med. 2004;23(19): 2937-2960.

38. Xie J, Liu C. Adjusted Kaplan-Meier estimator and log-rank test with inverse probability of treatment weighting for survival data. Stat Med. 2005;24(20): 3089-3110

39. Hernan MA, Brumback B, Robins JM. Marginal structural models to estimate the causal effect of zidovudine on the survival of HIV-positive men. Epidemiology. 2000; 11(5):561-570.

40. van der Wal WM, Geskus RB. IPW: an R package for inverse probability weighting J Stat Softw. 2011;43(13).

41. Hersh CM, Harris H, Conway D, Hua LH. Effect of switching from natalizumab to moderate- vs high-efficacy DMT in clinical practice. Neurol Clin Pract. 2020;10(6): e53-e65.

42. Austin PC. The performance of different propensity score methods for estimating marginal odds ratios. Stat Med. 2007;26(16):3078-3094.

43. Austin PC, Grootendorst P, Normand SL, Anderson GM. Conditioning on the propensity score can result in biased estimation of common measures of treatment effect: a Monte Carlo study. Stat Med. 2007;26(4):754-768.

44. Austin PC. The relative ability of different propensity score methods to balance measured covariates between treated and untreated subjects in observational studies. Med Decis Making. 2009;29(6):661-677.

45. Imbens GW. The role of the propensity score in estimating dose-response functions. Biometrika. 2000;87(3):706-710.

46. Imai K, van Dyk DA. Causal inference with general treatment regimes: generalizing the propensity score. J Am Stat Assoc. 2004;99(467):854-866.

47. Austin PC. Assessing the performance of the generalized propensity score for estimating the effect of quantitative or continuous exposures on survival or time-to-event outcomes. Stat Methods Med Res. 2019;28(8):2348-2367.

48. Austin PC. Assessing the performance of the generalized propensity score for estimating the effect of quantitative or continuous exposures on binary outcomes. Stat Med. 2018;37(11):1874-1894.

49. McCaffrey DF, Griffin BA, Almirall D, Slaughter ME, Ramchand R, Burgette LF. A tutorial on propensity score estimation for multiple treatments using generalized boosted models. Stat Med. 2013;32(19):3388-3414.

50. Rosenbaum PR. Observational Studies. Springer-Verlag; 2002.

\section{The AAN Is at Your Side}

When you're in the office, the AAN is at your side. The AAN is your \#1 resource to support you and your care team. Whether it's resources to help you and your staff provide the best care for your patients, ensure proper reimbursement, or maximize practice performance, the AAN is at your side. Access these resources today at AAN.com/practice. 


\section{Neurology}

\section{Applying Propensity Score Methods in Clinical Research in Neurology \\ Peter C. Austin, Amy Ying Xin Yu, Manav V. Vyas, et al. \\ Neurology 2021;97;856-863 Published Online before print September 9, 2021 \\ DOI 10.1212/WNL.0000000000012777}

This information is current as of September 9, 2021

\begin{tabular}{|c|c|}
\hline $\begin{array}{l}\text { Updated Information \& } \\
\text { Services }\end{array}$ & $\begin{array}{l}\text { including high resolution figures, can be found at: } \\
\text { http://n.neurology.org/content/97/18/856.full }\end{array}$ \\
\hline References & $\begin{array}{l}\text { This article cites } 46 \text { articles, } 4 \text { of which you can access for free at: } \\
\text { http://n.neurology.org/content/97/18/856.full\#ref-list-1 }\end{array}$ \\
\hline Citations & $\begin{array}{l}\text { This article has been cited by } 2 \text { HighWire-hosted articles: } \\
\text { http://n.neurology.org/content/97/18/856.full\#\#otherarticles }\end{array}$ \\
\hline Subspecialty Collections & $\begin{array}{l}\text { This article, along with others on similar topics, appears in the } \\
\text { following collection(s): } \\
\text { All Education } \\
\text { http://n.neurology.org/cgi/collection/all_education }\end{array}$ \\
\hline Permissions \& Licensing & $\begin{array}{l}\text { Information about reproducing this article in parts (figures,tables) or in } \\
\text { its entirety can be found online at: } \\
\text { http://www.neurology.org/about/about_the_journal\#permissions }\end{array}$ \\
\hline Reprints & $\begin{array}{l}\text { Information about ordering reprints can be found online: } \\
\text { http://n.neurology.org/subscribers/advertise }\end{array}$ \\
\hline
\end{tabular}

Neurology ${ }^{\circledR}$ is the official journal of the American Academy of Neurology. Published continuously since 1951, it is now a weekly with 48 issues per year. Copyright Copyright ( 2021 The Author(s). Published by Wolters Kluwer Health, Inc. on behalf of the American Academy of Neurology.. All rights reserved. Print ISSN: 0028-3878. Online ISSN: 1526-632X.

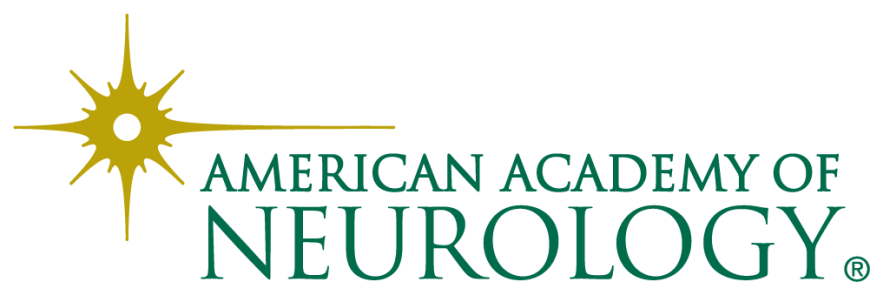

